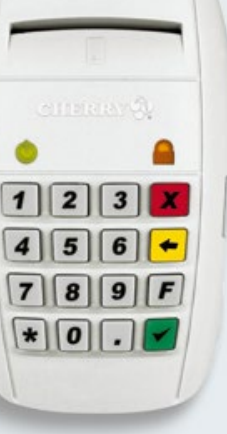

Cherry-Terminals auch mit neuer eGK kompatibel

Die Krankenkassen werden voraussichtlich im August mit der Ausgabe der neuen Generation (G2) der elektronischen Gesundheitskarte (eGK) starten. Wie der Anbieter von Kartenlesegeräten Cherry mitteilt, werden seine Terminals ST-2052, ST-1503 sowie der mobile SmartcardReader ST-1530 ab der Firmware-Version 3.2 keine Probleme mit der neuen Karte haben. Das ST-2052-Terminal sei dabei bis zur Einführung der Konnektoren die günstigste Alternative, es soll preislich der Austauschpauschale, die die Krankenkassen für die nicht mehr mit der Karte kompatiblen Geräte bereitstellen, entsprechen. Künftig vom Markt nehmen will Cherry hingegen seine eHealthTastatur G87-1504, das Gerät befinde sich am Ende des Lebenszyklus, heißt es. Cherry hat aber bereits ein Nachfolgemodell in der Zulassung, das zum Start der Online-Phase der eGK bereitstehen soll.

\section{Medistar startet \\ Abrechnungsservice}

Der Arztsoftware-Anbieter CGM Medistar bietet Praxen seit Kurzem auch einen Abrechnungsservice für die Privatliquidation. Neben dem Outsourcing von Rechnungsdruck und -versand, Eingangskontrolle und Mahnwesen beinhalte der neue Service einen GOÄ-LiveCheck. „Unsere Kunden werden gezielt in einem Expertentermin per Teamviewer bei einem ausgewählten Rechnungspaket zur GOÄ beraten. Dabei sieht die Praxis ganz konkret, wo bei ihr das Potenzial versteckt ist", so Alexander Boschuk, General Manager Geschäftsbereich CGM Medistar der CompuGroup Medical. Außerdem übernehme der Service den Schriftwechsel mit Patienten und Kostenträgern über strittige Ziffern, heißt es.

Rebekka Höh

\section{Digitaler Firmenstempel auch für} juristische Personen

Die eIDAS-Verordnung, die bereits im Sommer 2014 verabschiedet wurde, bietet noch mehr: Sie erlaubt es Unternehmen beziehungsweise Betrieben digitale Dokumente zentral mit einem elektronischen Siegel - also einer Art digitalem Firmenstempel - zu versehen. Damit könne der Siegelinhaber erstmals auch eine juristische Person sein, etwa eine $\mathrm{GmbH}$, erklärte Tatami Michalek, Geschäftsführer der secrypt GmbH im Gespräch auf der diesjährigen Gesundheits-IT-Messe conhIT in Berlin. Secrypt ist als Anbieter von Signaturanwendungen seit 2002 im Markt tätig. Laut Michalek ist es bislang so, dass qualifizierte Signaturzertifikate grundsätzlich einer natürlichen Person zugeordnet werden müssen. Qualifiziert bedeutet dabei, dass die E-Signatur auf einem zum Zeitpunkt der Erzeugung gültigen Zertifikat beruht und mit einer sicheren Signaturerstellungseinheit - etwa einer Signaturkarte - erzeugt wurde. Vor allem Krankenhäuser würden hoffen, dadurch ihre angestellten Ärzte im Alltag zu entlasten, so Michalek. Denn mit dem Siegel könnten die elektronisch archivierten Daten im Krankenhaus zentral signiert werden. Ebenso lassen sich elektronische Dokumente, die nach außen zum Beispiel an Patienten gehen - durch die Verwaltung mit dem digitalen Stempel versehen. Damit steigt der digitale Beweiswert. Das kann gerade auch für Medizinische Versorgungszentren oder große Berufsausübungsgemeinschaften interessant sein.

\section{QES unter dem E-Arztbrief} bleibt

Das Siegel ersetzt nicht die durch das E-Health-Gesetz geforderte qualifizierte elektronische Signatur (QES) des einzelnen Arztes unter dem E-Arztbrief. Diese ist zumindest dann nötig, wenn der Arzt im nächsten Jahr die Förderung von 55 Cent je signiertem E-Arztbrief einstreichen will. Wobei die Förderung nach dem ersten Entwurf der entsprechenden KBV-Richtlinie wohl gesplittet wird: 28 Cent soll der Sender, die übrigen 27 Cent der Empfänger des E-Arztbriefes erhal- ten. Das heißt, hier muss die Signatur in der Tat weiterhin vom einzelnen Arzt mithilfe einer Signatursoftware und seines elektronischen Arztausweises gesetzt werden. Allerdings haben die Anbieter von Signatursoftware - die meist direkt ins Praxisverwaltungssystem als Button integriert wird - längst die Stapelsignatur in ihre Systeme integriert. Dass die Signatur dann mit wenigen Mausklicks erledigt ist, demonstrierte auf der conhIT nicht nur Michalek. Am Stand der medisign $\mathrm{GmbH}$ war zu sehen, dass sich mit einmaliger PIN-Eingabe bis zu 254 Arztbriefe rechtssicher signieren lassen.

\section{Handy macht den Ausweis bald überflüssig}

Auch die Fernsignatur, die durch die EUVerordnung neu eingeführt wird, wird für die Anwendungen der elektronischen Gesundheitskarte (eGK) und Telematikinfrastruktur zunächst nicht zur Verfügung stehen. Spannend wird dieses Verfahren aber alle Mal: „Der Endanwender braucht hier weder Signaturkarte noch ein Lesegerät", so Michalek. "Ausgelöst wird die Signatur über das Mobiltelefon". Der private Signaturschlüssel werde hierbei zentral bei einem Vertrauensdiensteanbieter (Trust Center) und eben nicht mehr auf einer Signaturkarte gespeichert. Dabei sieht die Verordnung bislang ein sogenanntes Zwei-Faktor-Verfahren bei der Identifizierung der unterzeichnenden Person vor. Auf die Arztpraxis heruntergebrochen bedeutet das: Ein Teammitglied öffnet wie gewohnt das zu signierende Dokument und bestätigt den „Signieren-Button“. Daraufhin erhält das Trust Center eine Signaturanfrage und startet die Abfrage der Identität des Unterzeichners über das Mobiltelefon - etwa mittels Aufforderung zur TAN-, PIN- oder auch Fingerabdruckeingabe. Das Trust Center sendet wiederum einen Code zum Auslösen der Signatur an das Mobiltelefon des Anwenders. Der Praxismitarbeiter gibt diesen Code in den Computer ein und die Fernsignatur wird gesetzt. Ein Prozess, der nur wenige Sekunden dauert.

Rebekka Höhl 